\title{
Au-delà du baobab de Madame Livingstone
}

Réflexions sur le genre du voyage dans la France du XIX siècle

Beyond Mme Livingstone's baobab: considerations on the gender of travel in the $19^{\text {th }}$ century France

\section{Sylvain Venayre}

\section{(2) OpenEdition}

\section{Journals}

\section{Édition électronique}

URL : https://journals.openedition.org/clio/8082

DOI : 10.4000/clio.8082

ISSN : $1777-5299$

Éditeur

Belin

Édition imprimée

Date de publication : 15 décembre 2008

Pagination : 99-120

ISSN : $1252-7017$

Référence électronique

Sylvain Venayre, «Au-delà du baobab de Madame Livingstone », Clio. Histoire, femmes et sociétés [En ligne], 28 | 2008, mis en ligne le 15 décembre 2011, consulté le 22 avril 2022. URL : http://

journals.openedition.org/clio/8082 ; DOI : https://doi.org/10.4000/clio.8082 


\title{
Au-delà du baobab de Madame Livingstone. Réflexions sur le genre du voyage dans la France du XIX ${ }^{e}$ siècle
}

\author{
Sylvain VENAYRE
}

Fille du missionnaire écossais Robert Moffat, Mary Moffat rencontra au début des années 1840, à la mission de Kuruman fondée par son père dans le Bechuanaland, un jeune membre de la Société missionnaire de Londres, de huit ans son aîné et récemment arrivé en Afrique, David Livingstone. Elle l'épousa en 1844, le suivit dans un grand nombre de ses voyages dans l'intérieur du continent et eut avec lui six enfants, dont quatre vécurent. Après un retour en Angleterre pour se consacrer à l'éducation de ceux-ci, elle rejoignit son mari en Afrique, où elle mourut, sans avoir publié le moindre récit, à l'âge de quarante-et-un ans. Pour une de ses admiratrices françaises de la fin du XIX ${ }^{\text {e }}$ siècle, Marie Dronsart, il semblait qu'un tel destin eût réalisé les paroles bibliques de Ruth : «En quelque lieu que vous alliez, j'irai avec vous... La terre où vous mourrez me verra mourir et je serai ensevelie où vous le serez ». Marie Dronsart commentait :

Ce vœu suprême fut exaucé en la personne de Mme Livingstone, victime de la fièvre, tombée, en 1862, sur la scène de son sacrifice. Elle repose à Shoupanga, sur les bords du Zambèze, non loin de la côte de Mozambique, à l'ombre d'un gigantesque baobab; quelques pierres et une simple croix marquent la sépulture de l'hérö̈que compagne du missionnaire ${ }^{1}$.

1 Dronsart $1894: 4$. 
L'anecdote, célèbre, parait triplement révélatrice. D’abord, elle manifeste l'existence, au XIXe siècle, de femmes considérées comme de "grandes voyageuses", non seulement du fait de leurs pérégrinations, mais aussi du discours dont on les a entourées et dont témoigne un certain type de livres à succès : celui, précisément, de Marie Dronsart, réédité deux fois entre 1894 et la Grande Guerre - ou encore ceux d'aujourd'hui, dont la mode révèle l'intérêt du grand public pour les figures féminines les plus remarquables du passé, celles dont les exploits auraient prouvé l'inanité de la domination masculine ${ }^{2}$. Ensuite, la référence à madame Livingstone, sous la plume d'une Française, vient nous rappeler que ces "grandes voyageuses» du XIXe siècle furent principalement britanniques : le recueil de Marie Dronsart est peuplé d'Anglaises et un tel constat contribue à expliquer pourquoi l'historiographie française du voyage féminin est si pauvre, comparée à l'historiographie anglo-américaine ${ }^{3}$. Enfin, selon la morale du récit de la vie de madame Livingstone racontée par Marie Dronsart, la place de la femme dans l'histoire du voyage serait nécessairement secondaire d'une part, parce que les voyages de celles-ci ne seraient pas aussi importants que ceux des hommes, à commencer par ceux de David Livingstone; d'autre part, parce que les femmes, comme l'épouse du missionnaire et contrairement à son mari, auraient rapporté peu ou pas de récits de leurs périples. À l'image de madame Livingstone gisant sous son baobab, le souvenir des voyageuses du XIXe siècle serait enveloppé d'ombre.

On voudrait revenir ici sur cette image globale du voyage féminin dans la France du XIXe siècle, réduite à l'émergence de la figure de la grande voyageuse, au modèle des femmes britanniques, à la soumission aux voyages du mari et à l'absence de récit. Sans nier absolument ce

2 Voir par exemple Hodgson 2002, Lapeyre 2007, Lapierre \& Mouchard 2007.

3 Ce n'est bien sûr pas la seule raison, l'explication majeure résidant dans l'essor des gender et des colonial studies en Angleterre et aux États-Unis. Les études sur le voyage féminin sont du reste surtout des études littéraires. En France, le seul travail d'ensemble est Monicat 1996. Pour un bilan historiographique sur cette question, voir Melman dans ce numéro. 
qui précède, on voudrait suggérer les nuances d'une histoire qui, dans le cas français, reste encore largement à faire.

\section{L'émergence ambiguë des grandes voyageuses}

Partons ainsi de ce qui a d'abord retenu l'attention, dans la production destinée au grand public comme dans l'historiographie anglo-américaine: l'existence de grandes voyageuses, c'est-à-dire de femmes dont les voyages auraient la double caractéristique d'être insolites et d'avoir donné lieu à des récits contenant des informations inconnues jusqu'à elles, fût-ce dans leur forme. De tels cas, on le sait, sont rares et les raisons qui expliquent cela sont bien connues. Il y a d'abord la faible part consentie par l'édition, de façon générale, à l'écriture féminine, dans un temps qui postulait une relative contradiction entre femme et auteur ${ }^{4}$. Il y a, ensuite, le fait que les voyages relevaient souvent de professions fermées aux femmes, à commencer par celles de l'armée et de la marine. Enfin, les voyages dont il est question dans les différentes Histoires des voyages qui parurent, au XIXe siècle, entre l'Abrégé de La Harpe et les volumes de Jules Verne, étaient définis fondamentalement par leur capacité à accroître les connaissances ; en cela, ils se situaient dans la sphère d'un monde savant dont les femmes étaient exclues.

On trouverait, bien sûr, dès le début du XIXe siècle, des exceptions à cette règle - telles Mariana Starke ${ }^{5}$ ou Frances Trollope ${ }^{6}$. Dans

\section{Planté 1989.}

5 Mariana Starke (1761-1838) : anglaise, elle passa son enfance aux Indes. Après le retour de sa famille en Angleterre, elle partit vivre en Italie de 1792 à 1798. Elle y retourna en 1817-1819 et en 1838, où elle y mourut. Elle a écrit, outre des récits de voyages, des pièces de théâtre et des recueils de poésies. En 1832, le libraire Audin, éditeur des fameux guides Richard, en pirata la partie italienne pour publier un Guide classique du voyageur en Italie dont il indiquait, sur la page de couverture, le nom féminin de l'auteur.

6 Frances Trollope (1779-1863) : anglaise, elle s'installa aux États-Unis avec son mari en 1827 dans une communauté utopiste dont le projet échoua. À son retour en Angleterre, elle publia des récits sur l'Amérique. En 1837, un petit livre de vulgarisation des connaissances sur la géographie de l'Amérique, destiné aux 
l'ensemble, néanmoins, les femmes qui publiaient des récits de voyage avaient peu de chances de voir leur livre devenir une œuvre de référence, appréciée pour le sérieux de son contenu, l'exactitude des connaissances qui y étaient rapportées. Si le récit de voyage pouvait être, quoique de façon très marginale, un genre féminin, c'était à condition de se cantonner à l'expression des sentiments ressentis par la voyageuse, selon une logique tout à la fois récente dans le domaine du récit de voyage $^{7}$ et ancienne dans celui d'un genre jugé depuis longtemps plus en rapport avec la condition féminine: la correspondance privée ${ }^{8}$. En 1836, la comtesse de La Grandville publiait ainsi ses Lettres d'une voyageuse malade, présentées comme écrites pour le cercle de la famille et des amis, qu'elle n'aurait évidemment jamais publiées, dit son éditeur masculin ${ }^{9}$, sans l'insistance d'un père mourant. Un an plus tard, George Sand faisait paraittre, dans la Revue des Deux Mondes, ses propres Lettres d'un voyageur, dont la célèbre revendication esthétique ("Ne lis jamais mes lettres avec l'intention d'y apprendre la moindre chose certaine sur les objets extérieurs; je vois tout au travers de mes impressions personnelles $\left.{ }^{10} »\right)$ s'accordait certes à l'avènement du récit de voyage comme genre littéraire, mais également à la place traditionnellement réservée aux femmes dans l'écriture du voyage. Il convient d'avoir en tête cette hiérarchie des genres de récits pour ne pas surestimer la novation que semble parfois représenter l'écriture féminine du récit de voyage : l'enjeu est ici moins l'existence du récit que sa publication et son appartenance à l'univers savant.

De fait, il fallut attendre le milieu du siècle pour que le récit de voyage féminin pût être considéré comme susceptible d'apporter des connaissances nouvelles, leurs auteurs méritant alors le titre de

enfants, la citait aux côtés de Humboldt, Bonpland et Chateaubriand parmi les bonnes sources auxquelles l'auteur avait puisé.

Wolfzettel 1986 et 1996.

$8 \quad$ Planté 1998.

9 Voir Sauvé 2000 et Monicat 1994-1995.

10 Sand $1837: 271$. 
"grandes voyageuses » ${ }^{11}$. Des contenus de récits de voyages féminins devinrent recevables en termes de connaissance, renseignant désormais leurs lecteurs aussi positivement que les récits masculins. La première livraison du Tour du Monde, en 1860, comportait ainsi un récit inédit du voyage de l'Autrichienne Ida Pfeiffer à Madagascar ${ }^{12}$. Des articles de ce genre furent bientôt si nombreux dans la presse qu'en 1866, Richard Cortambert, secrétaire de la Société de géographie de Paris, put rassembler ceux que lui-même avait publiés dans divers périodiques pour en faire un livre intitulé Les Illustres Voyageuses. La matière en était des femmes qui non seulement avaient voyagé, mais encore avaient parcouru le monde "comme un champ d'étude», les récits qu'elles avaient rapportés prenant ainsi « une place estimable parmi les travaux les plus judicieusement composés $»^{13}$. Ce faisant, ce spécialiste de la vulgarisation du voyage savant avait bien conscience d'innover; il s'inquiétait des «esprits retardataires» qui pourraient ne pas admettre que l'on prît au sérieux les femmes qui voyageaient et adressait son livre «à tous ceux qui aiment le progrès et qui sont favorables aux pensées nouvelles $»^{14}$. D'autres ouvrages, volontiers écrits par des femmes, s'inscrivirent dans le sillage de celui de Richard Cortambert :

11 Une exception majeure à cette règle existait depuis la seconde moitié du XVIII siècle et les Lettres de Mary Montagu : les descriptions de harems. Lieu fermé aux hommes, le harem ne pouvait être décrit précisément que par des femmes et offrait ainsi, dans un XIXe siècle saisi par l'orientalisme, le moyen d'une reconnaissance savante du récit de voyage féminin. Sur cette question, voir Melman 1992 et Moussa 1995 : 175-198.

12 Ida Pfeiffer (1797-1858) : autrichienne, elle visita la Palestine et l'Égypte en 1842, puis la Scandinavie et l'Islande en 1845, ce qui donna lieu à deux récits de voyage publiés. En 1846, elle partit pour un tour du monde et revint à Vienne en 1848. Le récit de son voyage parut en 1850. En 1851, elle voyagea en Grande-Bretagne, puis à nouveau en Amérique du Sud. Elle passa ensuite huit mois dans l'archipel malais. Puis, elle voyagea en Australie, en Amérique encore, avant de revenir à Vienne en 1854. Un nouveau récit parut alors en 1856. En mai 1856, elle partit pour Madagascar, où elle fut impliquée dans une tentative de coup d'État et d'où elle fut expulsée. Elle mourut en 1858. Son récit de voyage à Madagascar parut à titre posthume. Voir Cahier d'images, figure 6.

13 Cortambert 1866 : préface.

14 Cortambert 1866 : préface. 
Les Exploratrices an XIXe siècle, de Charles Simond (1886), Les Voyagenses du XIX $X^{e}$ siècle, d'Amélie Chevalier (1888), en attendant Les Grandes voyagenses de Marie Dronsart. Ils inaugurèrent le genre du grand voyage féminin, que les années 1920 et 1930, avec la gloire posthume d'Isabelle Eberhardt, les récits d'Alexandra David-Néel et d'Ella Maillart ou les grandes figures de reporters que furent Andrée Viollis et Titaÿna, allait voir triompher ${ }^{15}$.

Pourtant, il convient ici de relativiser l'importance de ces cas isolés. Ni le début de reconnaissance sociale qui leur fut accordé, à partir de 1850, ni l'ambition progressiste et les allures de pionnier affichées par Richard Cortambert ne doivent nous conduire à surestimer le statut des grandes voyageuses avant les années 1920. Un triple dispositif, en effet, éloignait le lecteur du XIXe siècle de la tentation de considérer la destinée de voyageuse comme quelque chose de normal.

Ce lecteur, d'abord, était français; or les héroïnes qui étaient proposées à son admiration, dès lors qu'elles avaient fait seules le choix de leur voyage, étaient toutes étrangères. Le fait est assez remarquable pour devoir être signalé. Pour Richard Cortambert, il est certes des femmes «dont le cœur a palpité dès l'enfance à la lecture des découvertes et qui ont certainement envié la gloire des Colomb et des Cook $»^{16}$, mais parmi celles-ci, force est de constater qu'on ne trouve pas de Françaises ${ }^{17}$. Il n'y en a pas non plus dans les recueils ultérieurs, alors même que la poussée nationaliste de la fin du XIXe siècle conduisait le discours sur les grands voyageurs à faire la part belle aux héros de l'exploration française, de Brazza à Marchand. Dans tous ces livres, l'Autrichienne Ida Pfeiffer, la Hollandaise Alexine Tinné, bientôt la Russe Lydia Paschkov côtoyaient des voyageuses qui, pour la plupart, étaient anglaises, depuis Mary Montagu jusqu'à Marianne North en passant par Hesther Stanhope, Anna Brassey, Isabella Bird, Mary Barker, Anna Blunt ou Isabel Burton, et en attendant Mary

15 Voir Venayre $2002:$ 132-144 et Martin $2005:$ 292-297.

16 Cortambert 1866 : préface.

17 À l'exception du cas particulier de l'alpiniste madame d'Angeville. Sur la particularité des voyages des alpinistes françaises, voir Ottogalli-Mazzacavello 2006 . 
Kingsley ${ }^{18}$. Tous les auteurs constatèrent cette prééminence britannique, que Marie Dronsart expliquait de la façon suivante :

L'éducation des Anglaises, plus virile que la nôtre, la liberté d'action qu'on leur laisse dès leur première jeunesse et qui leur inspire la responsabilité d'elles-mêmes, la pratique de la marche, de l'équitation, des jeux et des exercices en plein air, les courses sur mer, le goût inné de leur race pour les voyages, tout cela réuni les prédispose à courir volontairement le monde ${ }^{19}$.

Les Américaines, pour les mêmes raisons, auxquelles s'ajoutait une supposée plus grande liberté de mœurs, pouvaient également faire de grandes voyageuses. Au début du $\mathrm{XX}^{\mathrm{e}}$ siècle, ce furent d'ailleurs les héroïnes américaines de Jack London, Frona Welse et Joan Lackland, qui fournirent aux lecteurs français les premières «aventurières »du roman d'aventure moderne ${ }^{20}$.

Un deuxième élément venait ensuite limiter la portée de l'existence de «grandes voyageuses" dans la seconde moitié du XIXe siècle. Les auteurs insistaient volontiers, surtout lorsqu'il s'agissait de Françaises, sur le fait que ces femmes illustres, à l'image de madame Livingstone, n'avaient pas choisi leur vocation. Si elles s'étaient lancées sur les grands chemins, c'était soit à la suite d'un événement tragique et imprévu, dont la figure majeure était le naufrage, à l'image de celui, matriciel, de madame Godin des Odonnais ${ }^{21}$; soit à cause du métier de leur mari, ce qui était le cas de Léonie d'Aunet, d'Adèle Hommaire de Hell, de Catherine de Bourboulon, de Jane Dieulafoy ou de Marie de Ujfalvy-Bourdon. Toutes ces femmes, ainsi que l'écrivait Richard Cortambert, ont « dit adieu à leur patrie avec regret; en quittant leurs foyers, c'est moins à un caprice qu'elles ont cédé, qu'un sacrifice

18 Sur celle-ci, voir Blunt 1994.

19 Dronsart $1894: 4$.

20 Héroïnes de Fille des neiges (1902, trad. 1933) et de L'Aventureuse (1911, trad. 1927). Voir Venayre 2006 : 146-149.

21 Madame Godin des Odonais avait fait naufrage sur l'Amazone en 1769. Son aventure fut souvent racontée dans la littérature de naufrage de la première moitié du XIXe siècle. 
qu'elles ont accompli » 22. Quels que soient leurs mérites et les légendes dont, par la suite, on a pu entourer certaines de leurs actions, rien ne pouvait enlever cette double dimension de hasard et de devoir qui avait présidé à leur départ.

Ainsi présenté, le groupe des «grandes voyageuses» de la seconde moitié du XIXe siècle était enfin encadré par un troisième dispositif discursif, qui rappelait à quel point le voyage n'était pas la condition naturelle de la femme. Le progressiste Richard Cortambert lui-même ne prétendait pas le contraire :

On dira que les femmes ne doivent pas suivre une voie diamétralement opposée à celle qui semble leur être tracée par la nature. Je ne réfuterai pas cet argument. Évidemment, si toutes les dames se mettaient à parcourir le monde, l'on serait en droit de se plaindre. La mère de famille, par exemple, a un autre rôle à accomplir que d'affronter les périls de lointaines pérégrinations ${ }^{23}$.

Un système de représentations cohérent écartait les femmes de la pratique du voyage. Certes les femmes étaient, ainsi que l'écrit Marie Dronsart, «curieuses »; et, à ce titre, le voyage pouvait les tenter ${ }^{24}$. De plus, leur extrême sensibilité garantissait l'intérêt de leurs récits, dont le charme venait d'abord de «la finesse de perception, la vivacité d'impression, la faculté d'assimilation» propres à leur sexe ${ }^{25}$. Néanmoins, le modèle de "l'ange du foyer» par lequel étaient alors résumés les devoirs d'épouse et de mère, leur inaptitude scientifique supposée, leur différence physique, qui faisait de la force, de l'intrépidité et de la hardiesse des caractères d'abord masculins, les éloignaient des grands chemins du globe.

Rien ne le montre mieux, a contrario, que la pure exception que fut, pour tous ces auteurs, Ida Pfeiffer. «Non seulement elle étonne par sa hardiesse, surprend par son incroyable énergie ", écrit Cortambert, «mais elle déroute, pour ainsi dire, tout raisonnement par la passion

22 Cortambert 1866 : préface. Voir Cahier d'images, figure 7.

23 Cortambert 1866 : préface.

24 Dronsart 1894 : préface.

25 Dronsart 1894 : préface. 
presque irréfléchie qui l'entraîne aux voyages. On ne trouve de vocation aussi franchement déterminée chez aucune femme $»^{26}$. Amélie Chevalier renchérit :

Les résultats scientifiques de ses explorations sont aussi curieux qu'intéressants. [...] elle ne prétend à aucun talent littéraire; elle ne tente pas de descriptions poétiques [...]. Par suite de cela, elle nous communique la conviction qu'elle n'a entrepris ces voyages extraordinaires ni sous l'empire de la vanité ni par l'ambition d'une vaine renommée, mais par goût et un insatiable désir d'accroître ses connaissances ${ }^{27}$.

Que ce soit donc par ses qualités physiques et morales - une intrépidité toute virile - ou par son écriture qui manifestait le goût masculin de la science, Ida Pfeiffer s'inscrivait dans le sillage de ces voyageurs savants qui, avant elle, avaient tous été des hommes. Comme l'écrit encore Amélie Chevalier,

quelqu'un a fait à son sujet cette observation, que si une nature douée d'autant de persévérance, de courage, de ténacité, avait eu à son service des forces masculines, l'histoire compterait un capitaine Cook ou un Magellan de plus ${ }^{28}$.

Hommage ambigu : les voyages d'Ida Pfeiffer en faisaient une femme si insolite qu'elle aurait dû être un homme, ce qui d'ailleurs, si la chose avait été possible, l'aurait conduit à faire des voyages plus remarquables encore. Le caractère exceptionnel de la "grande voyageuse », même lorsqu'il était pleinement admis, n'empêchait donc pas la logique opposant la femme et le voyage de demeurer active. Cette logique, que l'émergence de la figure de la "grande voyageuse », à partir des années 1850, ne remit pas vraiment en cause, il convient de l'étudier maintenant avec plus de précision.

26 Cortambert $1866:$ préface.

27 Chevalier 1888 : «Ida Pfeiffer».

28 Chevalier $1888:$ : Ida Pfeiffer». 


\section{Les motifs d'illégitimité du voyage féminin}

L'analyse d'une seconde conception du voyage est ainsi très éclairante : celle du voyage d'étude. L'utilité du voyage, ici, ne réside plus dans la capacité du voyageur à produire une connaissance absolument neuve - comme dans le cas du voyage savant et de l'exploration qui en devint, au XIXe siècle, la figure idéale. À l'image de ce qu'était le "Grand Tour » des élites du XVIII siècle, il s'agit de se déplacer pour aller vérifier sur place l'existence de quelque chose dont on a déjà, le plus souvent, une connaissance livresque ${ }^{29}$.

Une telle conception du voyage fut volontiers défendue par des femmes. Ainsi madame de Genlis, dès 1800, définissait-elle, sous la forme de dialogues utiles au jeune voyageur, tout l'intérêt du voyage d'étude. Même s'ils ne faisaient que reprendre des principes alors bien établis, les conseils de l'ancienne préceptrice des enfants du duc d'Orléans étaient présentés comme fondés sur son expérience personnelle; elle-même avait beaucoup voyagé, «même avant la révolution de France », en Angleterre et en Italie. À ses qualités de pédagogue elle ajoutait celles, jugées si féminines, de son style, évoquant «la sensibilité qui fait tout le mérite de mes écrits» ${ }^{30}$. Par la suite, de nombreuses femmes, dans la logique d'un temps qui leur confiait volontiers l'écriture des ouvrages pour les enfants, publièrent des récits de voyage imaginaires ayant le plus souvent pour but de faire apprendre la géographie. Elles citèrent d'ailleurs volontiers la phrase de madame de Genlis, selon laquelle «on pourrait faire de l'étude de la géographie une étude charmante $»^{31}$, l'adjectif renvoyant ici à une qualité éminemment féminine.

Dans toute cette littérature, cependant, il convient de remarquer que la part des femmes était l'œuvre de vulgarisation, la dimension savante demeurant l'affaire des hommes. Si Mme Guénard, baronne de Méré, publiait en 1818 Les Enfants voyageurs ou les petits botanistes, elle

29 Bertrand 2008.

30 Genlis $1800:$ II.

31 Voir par exemple Flesselles 1834: «Avis de l'éditeur» et Malte-Brun 1841 : «Préface». 
précisait bien que le livre était le fruit des cours suivis par l'auteur « au Jardin des Plantes sous M. Desfontaines ", savant naturaliste qui cautionna son travail après en avoir revu la partie botanique ${ }^{32}$. De la même façon, en 1820, le Petit Atlas de toutes les parties du monde de madame Tardieu-Denesle se refusait à apparaitre comme un ouvrage d'érudition, toute la partie savante du livre venant de MM. Mentelle et Malte-Brun ${ }^{33}$.

De plus, si ces ouvrages étaient parfois rédigés par des femmes, leurs personnages principaux, en revanche, étaient presque toujours des hommes. On peut ici distinguer trois grands modèles narratifs ${ }^{34}$. Le premier est celui, par exemple, des Jeunes Voyageurs en France, de madame de Flesselles, en 1834. L'intrigue était ainsi présentée: M. Dolmain, riche négociant tourangeau, père de six enfants, perd sa femme au moment où celle-ci lui donne une fille; dévoué depuis lors à l'éducation de ses enfants, il accepte un jour, pour les récompenser, de faire le récit des «aventures de voyages » que lui-même avait vécues dans sa jeunesse; le livre se présente ainsi comme un récit qui est l'occasion d'une description du territoire français ${ }^{35}$. Un second modèle narratif est celui d'Ernest et Fortunat ou les Jeunes Voyageurs en Italie, en 1837 : la famille Richard doit quitter Saint-Mandé pour un séjour d'un an dans le Midi, afin que madame Richard se remette d'une terrible maladie de poitrine; monsieur Richard en profite pour partir avec ses fils Fortunat et Ernest à la découverte de l'Italie à pied, tandis que son épouse demeure en convalescence, en compagnie de sa fille, à Nice; le livre est le récit du voyage du père et de ses fils, prétexte à une description de l'Italie ${ }^{36}$. Le troisième modèle est illustré par Les Jeunes Voyageurs en France ou Lettres sur les départements de G.-B. Depping, plusieurs fois réédité à partir de 1821 : Adolphe Verneuil veut épouser sa cousine Laure après avoir servi brillamment sous les drapeaux ; son

32 Guénard 1818 : « Avis du libraire-éditeur ».

33 Tardieu-Denesle 1820 : « Avertissement».

34 Indépendamment de la simple compilation de récits existant, sur le modèle de Bon 1821 ou Woilliez 1832.

35 Flesselles 1834 : « Avis de l'éditeur ». Voir aussi Malte-Brun 1841 : « Préface ».

36 Mirval 1837. Voir aussi Flesselles 1829. 
père y met la condition qu'il voyage d'abord à travers toute la France ; "voilà justement un voyage comme j'aurais voulu en faire un", s'exclame Laure, "puisque vous allez l'entreprendre, il faut que j'en profite »; le livre prend alors l'aspect d'une lettre par département, prétexte à une description historique, géographique et statistique de ceux-ci ${ }^{37}$.

Il existe, il est vrai, un quatrième modèle narratif, plus rare, lequel fait une place aux jeunes filles : celui, par exemple, du Trésor des voyages ou les petits correspondants, de Champagnac, en 1835. Dans ce cas, des enfants sont contraints à déménager ${ }^{38}$ - en l'occurrence, à cause du métier du père de famille. Dans ce cas seulement, sous l'influence d'événements extérieurs, une jeune fille peut être amenée à voyager. Mais, si elle tire de cette aventure du profit et même du plaisir, il n'en demeure pas moins qu'elle ne l'a pas organisée.

On pourrait multiplier les exemples de tels ouvrages. Tous ont les mêmes caractéristiques. D'abord, ces voyages sont faits par de jeunes gens : les héros de ces livres ont tous entre 13 et 18 ans. Ensuite, les femmes en sont toujours exclues - à tel point que, bien souvent, c'est la mort de la mère qui seule permet aux enfants et au père de réaliser enfin leur désir de voyage ${ }^{39}$. Enfin, et ce n'est pas le moins significatif, les femmes sont, à l'image de Laure, toujours intéressées par la connaissance tirée de ces voyages; mais elles ne peuvent y avoir accès que par des récits masculins, le voyage lui-même étant présenté comme une affaire d'hommes. De ce point de vue, l'impératif du voyage à

37 Depping 1821. Voir aussi Briand 1829.

38 Champagnac 1835. Voir aussi Briand 1827. C'est également le modèle narratif du Tour de la France par deux enfants, en 1877, mais les deux jeunes héros de madame Fouillée sont, classiquement, des garçons. Pour une analyse récente de cette littérature, voir Cabanel 2007.

39 Parmi d'autres titres: Alfred ou le jeune voyageur en France (1835), Oscar ou le jeune voyageur en Angleterre (1841), Firmin ou le jeune voyageur en Égypte (1843), Gustave ou le jeune voyageur en Espagne (1843), Arthur ou le jeune touriste (1847), Léon et Alice. Correspondance d'un jeune voyageur avec sa scur (1858). Sur le motif de la mort de l'épouse autorisant le voyage, voir entre autres le Guide des voyageurs et des curieux dans Paris (1822), L'Anacharsis indien (1838), Les Jeunes Voyageurs en Palestine (1843) ou Le Jeune Voyageur dans la Terre Sainte (1844). 
pied, recommandé par les pédagogues depuis la Renaissance et encore développé par Rousseau, renforçait la dimension masculine du voyage. Monsieur Richard explique tout cela de façon exemplaire à sa fille Hortense, qui voudrait bien partir en Italie avec Ernest, Fortunat et lui :

Il s'agit présentement de faire un petit voyage qui serait peut-être audessus des forces de ton sexe. Nous voyagerons quelquefois en voiture, mais le plus souvent il nous faudra aller en bons piétons; car c'est le véritable moyen de voir bien et d'examiner à l'aise tout ce qu'on rencontre de curieux et d'intéressant. Une jeune personne, délicate comme tu l'es, s'exposerait à compromettre sa santé dans de pareilles courses, où le plaisir s'achète souvent au prix de la fatigue et de mille privations de tous genres. D'ailleurs l'éducation des femmes n'a nullement besoin de l'expérience des voyages pour se perfectionner. L'économie domestique, le gouvernement intérieur de la famille, les qualités douces et aimables qui font l'apanage des épouses et des mères, les talents qui les aident à faire le charme de la société, tout cela s'acquiert facilement au foyer paternel ; il n'en est pas de même de l'éducation des jeunes gens. Il importe qu'ils voyagent un peu, afin d'acquérir des notions exactes sur les hommes et sur les choses ${ }^{40}$.

Si elles ne leur sont pas d'une utilité immédiate, les connaissances que l'on peut rapporter d'un voyage ne sont pas formellement interdites aux femmes - après tout, celles-ci pouvaient consacrer leurs loisirs à l'étude de l'art comme à celle de la botanique, que le voyage favorisait. Mais le déplacement lui-même leur était déconseillé. Les raisons tenaient entre autres, bien sûr, à des considérations de nature physiologique. Les médecins soulignaient volontiers que les longs voyages, toujours pénibles, l'étaient particulièrement pour les femmes ${ }^{41}$. Les chaos des diligences, puis les trépidations du chemin de fer leur étaient plus dangereux qu'aux hommes. Il en allait ainsi également de certains climats. En 1865, le Guide du voyageur en Algérie des frères Garnier notait par exemple que, là-bas, « la femme éprouvera du côté de la menstruation des modifications dont elle doit être

40 Mirval $1837: 12$.

$41 \quad$ Voir par exemple Decaisne 1864. 
avertie » et rappelait que « le climat prédispose à l'avortement la femme qui arrive grosse en Algérie, ou qui le devient peu après son arrivée » ${ }^{42}$. Dans l'ensemble, sa supposée fragilité de constitution rendait à la femme tous ses déplacements plus compliqués ; là pourtant n’était pas l'essentiel.

L'essentiel résidait, d'une part, dans l'inutilité fondamentale, pour des femmes auxquelles était destinée la bonne tenue du foyer, des connaissances tirées du voyage. À quoi s'ajoutait, d'autre part, le soupçon d'immoralité pesant sur la femme en voyage. Rappelons que ce discours sur le voyage d'agrément s'adressait à une certaine élite sociale: celle qui, précisément, pouvait s'autoriser de tels voyages. L'idéal féminin qu'il mettait en scène était donc également celui de ces élites pour lesquelles la dignité de l'épouse et de la fille manifestait celle du mari et du père. Selon cette logique, il était difficile pour une femme d'avoir plus mauvaise réputation qu'en voyageant seule. Toute une littérature en témoigne, à l'image de cet Amour au grand trot qui, en 1820, recommandait aux jeunes hommes de se méfier des « gaudrioleuses de messageries »:

Elles n'ont pas d'autre domicile que les voitures publiques: elles se plantent dans l'intérieur ou le cabriolet, sans papiers, sans argent ; laissent couler insensiblement leurs schals [sic] pour découvrir, sans affectation, l'élégance de leur taille et les voluptueux contours de leur gorge; elles ôteront encore leurs gants, si elles ont la main jolie ; riront sans motifs pour montrer leurs belles dents ; auront grand soin, surtout en descendant, de se trousser un peu haut, afin de laisser voir le beau présage de leurs mollets... Et tout cela, se disent-elles d'avance, équivaut à une lettre de change tirée à vue sur le premier gobe-mouche qui va payer à dîner, à souper, à déjeuner... qui payera tout enfin ${ }^{43}$.

À ce sujet, les témoignages sont innombrables : on citera seulement celui de Flora Tristan scandalisée, en 1835, par la façon dont les

42 Fillias $1865: 119$.

43 Vélocifère 1820: «Discours préliminaire ». L'avènement des chemins de fer déporta ce discours dans le cadre du compartiment ou de la gare. Sur ce processus d'exclusion de la femme du voyage en chemin de fer, voir Sauget 2005 : 380 et sq. 
femmes seules sont traitées dans les hôtels ou les villes d'eaux - et souhaitant remédier à cela par la fondation d'une "Société pour les Femmes Étrangères ${ }^{44}$. En fait, tout se passe comme si le voyage, par la rupture qu'il introduit dans la vie quotidienne, impliquait nécessairement la possibilité de la passade, l'éventualité de l'aventure. En 1841, une des illustrations accompagnant la Physiologie du voyageur montrait, dans un compartiment de chemin de fer, un homme se jetant sur une femme à l'occasion d'un de ses «noirs tunnels, où l'œil ne voit rien et où par conséquent l'imagination a le droit de faire ses évocations les plus hardies ${ }^{45} »$. De tels fantasmes ne s'accordaient pas avec la figure de "l'ange du foyer»; les hommes qui les rapportaient les peuplaient d'intrigantes, de servantes et de putains. Mais les dames qui souhaitaient s'adonner aux délices du voyage d'agrément en étaient comme contaminées.

\section{Les femmes en voyage}

Or ces dames étaient nombreuses : on ne saurait trop insister sur ce point. L'ambiguité de la figure de la «grande voyageuse » (et la relative rareté des cas qui l'illustraient), de même que le ressassement des motifs d'illégitimité du voyage féminin, ne doivent pas faire oublier que les femmes du XIX siècle voyagèrent massivement et ce, indépendamment des obligations que pouvaient leur imposer les devoirs de famille.

Ainsi, dans un siècle pendant lequel le catholicisme fut de plus en plus une affaire de femmes, les pèlerinages se féminisèrent ${ }^{46}$. Les femmes étaient très nombreuses au sanctuaire de Sainte Anne d'Auray par exemple ${ }^{47}$, et les pèlerins qui se rendaient auprès du curé d'Ars, au milieu du siècle, pour plus de la moitié d'entre eux, étaient des

44 Tristan $1835: 67$.

45 Alhoy $1841: 76$.

46 On n'évoquera pas ici la question du rapport entre voyage et pratique missionnaire, pourtant fascinante dans le cas des femmes. Sur ce point, voir l'article de Nicole Pellegrin dans ce même numéro.

47 Lagrée 1999: 133. Lagrée souligne aussi le rôle joué par l'invention de la voiture d'enfant dans la féminisation des pèlerinages au XIX ${ }^{\mathrm{e}}$ siècle. 
pèlerines ${ }^{48}$. Certes, plus le pèlerinage était lointain, moins on $y$ comptait de femmes. Lorsque, dans les années 1850, l'Euvre des pèlerinages en Terre-Sainte de la Société Saint-Vincent-de-Paul organisa ses premiers voyages à Jérusalem, seuls des hommes y participèrent. Les préventions contre le voyage féminin jouèrent dans ce cadre aussi. Il n'empêche que la vogue des pèlerinages nationaux, notamment à partir du moment de l'achèvement du réseau ferré principal vers 1860, s'est traduite par de nombreux déplacements féminins, que légitimaient tout ensemble leur but pieux et la norme imposée par le siècle d'effectuer ces voyages en groupe, le plus souvent sous la direction d'un prêtre ${ }^{49}$.

Il faut en dire autant des voyages occasionnés par la réinvention des stations thermales et par l'invention des stations balnéaires et hivernales. Réservés au début du XIXe siècle à une élite sociale, ils étaient le plus souvent accomplis en couple ou en famille et, de ce fait, centrés sur la figure de l'épouse. C'étaient les maux de celle-ci qu'on devait d'abord aller soigner, ainsi que le rappelle, dans les petits ouvrages destinés aux enfants évoqués plus haut, le motif récurrent de la mère malade, conduisant la famille dans le Midi, sur les bords de l'Océan ou dans les villes d'eaux. Deux des grandes figures féminines du voyage au XIXe siècle furent, à n'en pas douter, la valétudinaire et la convalescente, les Lettres d'une voyageuse malade de la comtesse de La Grandville, déjà citées, ayant ici encore valeur d'exemple.

Le voyage de santé, toutefois, évolua progressivement en direction du séjour ou de l'excursion touristique, diffusant des pratiques que le XVIII e siècle avait déjà connues et qu'avait illustrées, parmi d'autres, madame de Genlis. Dans ce souci de mêler guérison et désir d'agrément, les contemporains notèrent d'ailleurs le rôle joué par les "grandes dames». On rappela vite que c'était l'engouement de la duchesse de Berry pour Dieppe qui avait fait le succès de la première station balnéaire française, en attendant que la reine Amélie, puis l'Impératrice Eugénie n'assurassent la réussite d'autres lieux de

48 Boutry \& Cinquin 1980.

49 Venayre 2009. 
villégiature. De même, on nota la liberté de ton et de manières caractéristiques des «Paris d'été ». La Physiologie du voyageur de 1841, toujours égrillarde, ironisait ainsi sur ces honnêtes époux qui n'hésitaient pas à déposer leurs femmes dans les bras de beaux «baigneurs » à moitié nus qui les portaient dans les vagues, alors que des effleurements bien plus discrets, aperçus à Paris, auraient paru « un attentat aux mœurs ${ }^{50}$. De façon générale, les relations entre les sexes étaient en partie réaménagées au sein des petites sociétés de ces différentes stations - ce dont témoignent d'ailleurs les codes de savoirvivre qui, sous la monarchie de Juillet et l'Empire notamment, mettaient en garde les dames du monde contre de tels lieux, où plus d'une réputation se seraient perdues ${ }^{51}$.

Ces voyages d'agrément touchèrent, au fur et à mesure de l'avancée du siècle, de plus en plus de femmes. L'amélioration des moyens de transport joua ici un rôle considérable. Dès les années 1820, le développement de la navigation à vapeur autorisa ainsi des voyages - par exemple sur la Seine, entre Paris et le Havre- que les compagnies destinaient aux femmes autant qu'aux hommes ${ }^{52}$. Dans la seconde moitié du XIX ${ }^{\mathrm{e}}$ siècle, l'influence du chemin de fer parut décisive aux contemporains, alors que se multipliaient les études cherchant à prouver l'inocuité du train sur la santé53 et qu'une ordonnance ministérielle du 1er mars 1861 stipulait que «les femmes seules ont le droit d'exiger qu'on les mettre dans une voiture qui leur est spécialement destinée, surtout en première et en seconde classe, et une étiquette, portant le mot Dame ou Réservé, posée sur le wagon ${ }^{54}$. Le docteur Decaisne, en 1864, se félicitait d'une telle évolution :

$50 \quad$ Alhoy $1841: 118$.

51 Voir par exemple Drohojowska 1860 : 135-137.

52 «Le voyage par le bateau à vapeur est très agréable pour les dames; non seulement elles ne se fatiguent point, mais elles ont l'avantage de ne pas déranger leur toilette » (Guide du voyageur à Saint-Cloud, Paris/Saint-Cloud, 1826).

53 Voir par exemple Pietra Santa 1861.

54 Manuel $1861: 48$. 
Nous ne sommes plus au temps où madame de Sévigné faisait, pour se rendre aux eaux de Bourbon l'Archambault, plus de préparatifs qu'on en fait aujourd'hui pour se rendre aux établissements thermaux de l'Allemagne ou de notre extrême Midi; aller aux eaux est une partie de plaisir, une simple distraction, une occasion de montrer ses belles toilettes, pour les dames ${ }^{55}$.

Le mot d'ordre de la démocratisation du voyage, dans les années 1860, concerna ainsi les femmes. L'enjeu majeur fut désormais l'hôtellerie car, comme on pouvait encore le lire dans une édition de 1885 d'un guide Baedeker, «en voyageant avec des dames [...] on choisira toujours un des premiers hôtels; un homme seul se tire d'affaire partout ${ }^{56}$. La création de bagages et de garde-robes de voyage spécialement réservés aux femmes s'accompagna bientôt de l'invention, par les agences spécialisées bâties sur le modèle proposé par Thomas Cook, d'excursions touristiques auxquelles les femmes pouvaient participer sans s'exténuer. À la fin des années 1890, l'agence parisienne Desroches se présentait même comme spécialisée dans l'organisation de voyages de groupes pour les femmes ${ }^{57}$.

L'essor des voyages d'agrément au XIXe siècle concerna ainsi, au premier chef, les femmes. Nul doute qu'elles furent nombreuses à considérer bénéficier alors de l'élargissement des horizons, des vertus de la rencontre et de tout ce que le siècle avait défini, de plus en plus précisément, comme les vertus du voyage. Nul doute non plus qu'elles témoignèrent par l'écriture des connaissances acquises et des impressions ressenties pendant leurs voyages, s'appropriant une tradition d'écriture qui avait été encouragée, depuis la Renaissance, auprès des jeunes hommes ${ }^{58}$. Correspondances privées et journaux intimes regorgent ainsi de bribes de récits de voyages qui, pour ne pas avoir la visibilité ni le statut savant du récit masculin publié, n'en témoignent pas moins aujourd'hui, pour l'historien, des formes prises par la diffusion du voyage féminin au XIX ${ }^{\mathrm{e}}$ siècle.

55 Decaisne 1864 : XIV.

56 Baedeker 1885 : préface.

57 Vajda $2005: 160$.

$58 \quad$ Stagl 1994. 
Si un tel constat doit certainement être un encouragement à l'étude de ces récits encore trop peu connus, il ne doit toutefois pas masquer l'ambiguité majeure qui accompagna l'essor du voyage féminin, sous toutes ses formes, jusqu'à la Grande Guerre - ambiguité dont témoigne la fortune, à la Belle Époque, du «voyage de noces». Cette invention est certainement la plus significative du XIX ${ }^{e}$ siècle dans le domaine du voyage féminin et il est curieux qu'elle n'ait pas davantage fait l'objet d'études ${ }^{59}$. Timidement émergente dans les années 1820, la pratique nouvelle a gagné son nom dans les années 1870 avant de triompher à partir des années 1880, imposant l'idée que toute dame mariée devait avoir fait au cours de sa vie, au moins une fois, un beau voyage. Pourtant, étroitement lié au développement remarquable, à la fin du XIXe siècle, du voyage d'agrément, le voyage de noces n'en imposa pas moins une conception du voyage qui, d'une part, s'accordait profondément à la condamnation du voyage des femmes seules et qui, d'autre part, centrait les découvertes de la voyageuse sur le corps de son époux et le plaisir sexuel, au détriment des pays traversés et des peuples rencontrés, dont les représentations étaient d'ailleurs très strictement codifiées. Davantage: en associant le souvenir du voyage à celui du mariage, le voyage de noces laissait entendre qu'un tel voyage de l'épouse serait, à tous les sens du terme, unique - c'est-à-dire qu'il n'y en aurait pas d'autres. La maternité, qui est le futur du mariage, éloignerait définitivement les femmes des grands chemins : dès 1883, Guy de Maupassant proposa, dans Une vie, une terrible variation sur ce voyage féminin d'emblée condamné à n'être qu'un beau souvenir. Certes, on l'a dit, les femmes voyagèrent en réalité beaucoup; le triomphe de la pratique du voyage de noces n'en manifesta pas moins celui d'une norme contraignante.

Mary Moffat, épouse Livingstone, accompagna son mari au lendemain de leur mariage de 1844, dans des expéditions qui avaient peu à voir avec cette pratique du voyage « à la façon anglaise » qui allait devenir, en France, le voyage de noces. Depuis le milieu du XIX ${ }^{e}$ siècle,

59 Voir Venayre 2007. 
ceux qui se sont penchés sur le cas des "grandes voyageuses» voudraient que celles-ci témoignent de quelque chose, selon une démarche intellectuelle d'ailleurs ambiguë. Les "grandes voyageuses", en effet, oscillent entre le statut d'avant-garde annonçant les évolutions à venir et celui d'exceptions magnifiques dont l'étude, en creux, manifesterait la norme d'une époque. Enterrée sous son baobab, de quoi madame Livingstone témoignerait-elle? Serait-elle l'exception dans un siècle où les femmes, tout particulièrement les Françaises, voyageaient peu? Certainement pas. Non seulement les voyages de Mary Livingstone ne sont pas plus représentatifs de ceux des femmes de son temps que les voyages de David ne le sont de ceux des hommes; mais surtout les femmes voyagèrent en nombre au XIXe siècle, de plus en plus avec le temps - et ce, quelles que furent les considérations physiologiques sur la nature féminine, au point même de faire de la femme malade une des grandes figures du voyage au XIXe siècle. Annoncerait-elle le temps à venir du voyage des femmes seules ? Pas davantage. Mary Livingstone ne voyagea qu'en compagnie de son mari, selon une logique qui ordonnait alors non seulement les pratiques des "grandes voyageuses", mais aussi celles des dames en voyage d'agrément, pour lesquelles le siècle inventa la norme si précisément codifiée du voyage de noces - ainsi que celles des pèlerines, dont les déplacements se faisaient en groupe et sous la direction d'un prêtre. En cela, la femme du missionnaire Livingstone ne s'écartait pas du dogme de l'illégitimité du voyage féminin solitaire. Jouerait-elle alors un rôle dans l'avènement d'un récit de voyage féminin original ? Encore moins. Mary Livingstone n'ayant rien publié, elle ne put lutter contre le grand partage du siècle qui, s'il accordait la «sensibilité » féminine au prestige nouveau de l'écriture de l'impression, tâchait de cantonner les femmes dans la sphère de la correspondance privée et du journal intime. Aussi est-ce probablement de l'exhumation de ces sources enfouies, plus que de l'étude des « grandes voyageuses ", que l'on doit espérer, dans l'histoire du voyage des femmes au XIX siècle, des résultats neufs. Nous avons tâché de résumer ici les normes, proposées par le siècle, du voyage féminin. L'essentiel reste à faire : l'analyse de ces lettres et de ces carnets dans lesquels se sont manifestées les 
modalités, tout à la fois individuelles et socialement distribuées, de l'appropriation de ces normes.

\section{Bibliographie}

AlHoy Maurice, 1841, Physiologie du voyageur, Paris, Aubert et Cie.

BAEDEKER Karl, 1885, Belgique et Hollande. Manuel du voyageur, Leipzig, Baedeker.

Bertrand Gilles, 2008, Le Grand Tour revisité. Pour une archéologie du tourisme (Le voyage des Français en Italie, milieu XVIII siècle-début XIXe siècle), Rome, École française de Rome.

BuUnt Alison, 1994, Travel, Gender and Imperialism: Mary Kingsley and West Africa, New York, Guilford Press.

Bon Elisabeth de, 1821, Voyageur moderne, Paris, Eymery.

Boutry Philippe \& Michel CINQUIN, 1980, Deux pèlerinages au XIXe siècle : Ars et Paray-leMonial, Paris, Beauchesne.

BRIAND P.-C., 1827, Les Jeunes voyageurs en Europe, Paris, Thiériot.

—, 1829, Les Jeunes Voyageurs en Asie, Paris, Hivert.

CABAnel Patrick, 2007, Le Tour de la nation par des enfants. Romans scolaires et espaces nationaux (XIXe-XX' siècles), Paris, Belin.

Champagnac J.-B., 1835, Le Trésor des voyages ou les petits correspondants, Paris, Lehuby.

CheVAlier Amélie, 1888, Les Voyageuses au XIXe siècle, Tours, Mame.

CORTAMBERT Richard, 1866, Les Illustres voyageuses, Paris, Maillet.

DECAISNE Dr. Émile, 1864, Guide médical et hygiénique du voyageur, Paris, Albessard.

Depping Georges-Bernard, 1821, Les Jeunes voyageurs en France ou Lettres sur les départements, Paris, Ledoux.

DrohojowsKa Comtesse, 1860, De la politesse et du bon ton ou Devoirs d'une femme chrétienne dans le monde, Paris, Sarlit.

DronsarT Marie, 1894, Les Grandes Voyageuses, Paris, Hachette.

Fillias Achille, 1865, Nouvean guide général du voyageur en Algérie, Paris, Garnier.

Flesselles Madame de, 1829, Les Jeunes voyageurs dans Paris ou les Tablettes de Jules, Paris, Locard et Davi.

—, 1834, Les Jeunes voyageurs en France, Paris, Lehuby.

GENLIS Madame de, 1800, Le Voyageur. Ouvrage utile à la jeunesse et aux étrangers, Berlin, La Garde.

GUÉNARD Madame, 1818, Les Enfants voyageurs ou les petits botanistes, Paris, Eymery.

Hodgson Barbara, 2002, Les Aventurières. XVII ${ }^{e}$-XIX ${ }^{e}$ siècles. Récits de femmes voyageuses, Paris, Seuil.

LAGRÉE Michel, 1999, La Bénédiction de Prométhée. Religion et technologie. XIXe-XXe siècles, Paris, Fayard.

LAPEYRE Françoise, 2007, Le Roman des voyageuses françaises (1800-1900), Paris, Payot.

LAPIERRE Alexandra \& Christel MOUCHARD, 2007, Elles ont conquis le monde: les grandes aventurières. 1850-1950, Paris, Arthaud. 
Malte-Brun Victor, 1841, Les Jeunes Voyageurs en France, Paris, Lehuby.

MARTIN Marc, 2005, Les Grands reporters. Les débuts du journalisme moderne, Paris, Audibert.

Manuel complet du voyageur sur les chemins de fer français, 1861, Paris, Ledoyen.

Melman Billie, 1992, Women's Orients. English Women in the Middle East. 1718-1918. Sexuality, Religion and Work, Ann Arbor, University of Michigan Press.

Mirval C.-J., 1837, Ernest et Fortunat ou les jeunes voyageurs en Italie, Paris, Lehuby.

Monicat Bénédicte, automne-hiver 1994-1995, «Problématique de la préface dans les récits de voyage au féminin au XIXe siècle», Nineteenth-Century French Studies, 23, p. $59-71$.

—, 1996, Itinéraires de l'écriture au féminin. Voyageuses du XIXe siècle, Amsterdam, Rodopi.

Moussa Sarga, 1995, La Relation orientale. Enquête sur la communication dans les récits de voyage en Orient (1811-1861), Paris, Klincksieck.

Ottogalli-Mazzacavello Cécile, 2006, Femmes et alpinisme (1874-1919): un genre de compromis, Paris, L'Harmattan.

Pietra Santa Dr. Prosper de, 1861, Chemins de fer et santé publique. Hygiène des voyageurs et des employés, Paris, Hachette.

Planté Christine, 1989, La Petite Sxur de Balzac. Essai sur la femme auteur, Paris, Seuil.

—, (dir.), 1998, L'Epistolaire, un genre féminin ?, Paris, Champion.

SAND George, 1837, Lettres d'un voyageur (1971), Paris, GF-Flammarion.

SAuget Stéphanie, 2005, A la recherche des Pas Perdus. Dans la matrice des gares parisiennes (1837-1914), thèse de doctorat d'histoire, Université de Paris-I.

SAUVÉ Rachel, 2000, De l'éloge à l'exclusion. Les femmes auteurs et leurs préfaciers au XIXe siècle, Presses universitaires de Vincennes.

STAGL Justin, 1994, A History of curiosity : the theory of travel. 1550-1800, Shur, Harwood academic publiphobies.

TArdieu-Denesle Madame, 1820, Petit Atlas de toutes les parties du monde, Paris, TardieuDenesle.

Tristan Flora, 1835, Nécessité de faire un bon accueil aux femmes étrangères, Paris, Delaunay.

VajDA Joanne, 2005, Paris : rendez-vous cosmopolite. Du voyage élitaire à l'industrie touristique. 1855-1937, thèse de doctorat d'histoire, EHESS.

VÉLOCIFÈRE, 1820, L'Amour au grand trot ou la gaudriole en diligence, Paris.

Venayre Sylvain, 2002, La Gloire de l'aventure. Genèse d'une mystique moderne. 1850-1940, Paris, Aubier.

—, 2006, Rêves d'aventures. 1800-1940, Paris, La Martinière.

—, Juin 2007, « Le Temps du voyage de noces », L'Histoire, 321, p. 56-61.

—, 2009, "Catholicisme, romantisme et tourisme: le pèlerin en France de 1815 à 1870 », in Dominique Dinet (éd.), Le Voyage, Strasbourg, Presses universitaires de Strasbourg.

WoILLIEZ Catherine, 1832, Vies et aventures des grands voyageurs, Limoges, Ardant.

WolfzETTEL Friedrich, 1986, Ce désir de vagabondage cosmopolite. Wege und Entwicklung des französischen Reiseberichts im 19. Jabrbundert, Tübingen, Max Niemeyer Verlag, 1986

—, 1996, Le Discours du voyageur. Le récit de voyage en France du Moyen Age au XVIII siècle, Paris, PUF. 